\title{
Phenomenology of a flow around a circular cylinder at sub-critical and critical Reynolds numbers
}

Cite as: Phys. Fluids 28, 074101 (2016); https://doi.org/10.1063/1.4954655

Submitted: 23 December 2015 . Accepted: 10 June 2016 . Published Online: 01 July 2016

Alessandro Capone, Christian Klein, Fabio Di Felice, and (D) Massimo Miozzi

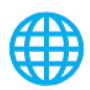

\section{ARTICLES YOU MAY BE INTERESTED IN}

Unsteady forces on a circular cylinder at critical Reynolds numbers

Physics of Fluids 26, 125110 (2014); https://doi.org/10.1063/1.4904415

Numerical studies of flow over a circular cylinder at $\mathrm{Re}_{D}=3900$

Physics of Fluids 12, 403 (2000); https://doi.org/10.1063/1.870318

Experimental and numerical studies of the flow over a circular cylinder at Reynolds number 3900

Physics of Fluids 20, 085101 (2008); https://doi.org/10.1063/1.2957018

\section{AlP Advances Fluids and Plasmas Collection}




\title{
Phenomenology of a flow around a circular cylinder at sub-critical and critical Reynolds numbers
}

\author{
Alessandro Capone, ${ }^{1, \text { a) }}$ Christian Klein, ${ }^{2}$ Fabio Di Felice,${ }^{1}$ \\ and Massimo Miozzi ${ }^{1, b)}$ \\ ${ }^{1}$ CNR-INSEAN, Via di Vallerano 139, 00128 Rome, Italy \\ ${ }^{2}$ German Aerospace Center (DLR), Bunsenstrasse 10, 37073 Göttingen, Germany
}

(Received 23 December 2015; accepted 10 June 2016; published online 1 July 2016)

\begin{abstract}
In this work, the flow around a circular cylinder is investigated at Reynolds numbers ranging from 79000 up to 238000 by means of a combined acquisition system based on Temperature Sensitive Paint (TSP) and particle velocimetry. The proposed setup allows simultaneous and time-resolved measurement of absolute temperature and relative skin friction fields onto the cylinder surface and near-wake velocity field. Combination of time-resolved surface measurements and planar near-field velocity data allows the investigation of the profound modifications undergone by the wall shear stress topology and its connections to the near-field structure as the flow regime travels from the sub-critical to the critical regime. Laminar boundary-layer separation, transition, and re-attachment are analyzed in the light of temperature, relative skin friction maps, and Reynolds stress fields bringing about a new perspective on the relationship between boundary layer development and shear layer evolution. The fast-responding TSP employed allows high acquisition frequency and calculation of power spectral density from surface data. Correlation maps of surface and nearwake data provide insight into the relationship between boundary-layer evolution and vortex shedding. We find that as the Reynolds number approaches the critical state, the separation line oscillations feature an increasingly weaker spectrum peak compared to the near-wake velocity spectrum. In the critical regime, separation line oscillations are strongly reduced and the correlation to the local vorticity undergoes an overall decrease giving evidence of modifications in the vortex shedding mechanism. Published by AIP Publishing. [http://dx.doi.org/10.1063/1.4954655]
\end{abstract}

\section{INTRODUCTION}

The flow around a circular cylinder is considered as one of the classical problems in bluff-body aero- and hydro- dynamics and a vast amount of experimental data have been collected over the last decades (Zdravkovich, 1997 and Williamson, 1996). Despite its apparently simple configuration, the underlying flow phenomena are very complex and show a strong dependence on Reynolds number. In particular, the flow regime may be sorted in three main phases, depending on the flow region where transition from laminar to turbulent flow occurs: (i) the cylinder's wake, (ii) the shear layers, and (iii) the boundary layer (Zdravkovich, 1997 and Williamson, 1996). The transition-in-boundary layer state is marked by a dramatic drop of the total drag and is characterized by the transition through various sub-states: pre-critical, one-bubble, and two-bubble regime (Zdravkovich, 1997). The evolution of these flow regimes is quite sensitive to variations of the Reynolds number and boundary conditions.

In the current work, we focus on flow states ranging from the sub-critical, transition-in-shearlayers regime up to the critical regime. These flow states are reported to be associated with a

\footnotetext{
a) Electronic mail: alessandro.capone@uniud.it

b) Electronic mail: massimo.miozzi@cnr.it.
} 
range of Reynolds numbers (20-40k $<R e<300-340 \mathrm{k}$ ) which is often encountered in mechanical and chemical engineering applications. Furthermore, as regards the critical regime, applications for which drag reduction is a key factor represent a common field of interest.

The flow around circular cylinders has been the focus of a vast amount of experimental work over the last decades. Recently, in the works by Perrin et al. (2007) and Perrin et al. (2008) time-resolved particle image velocimetry has been employed to investigate the near-wake structure at high, sub-critical Reynolds numbers. The authors relied on phase averaging and modal decomposition to analyse the effect of the coherent structures motion on the structural properties of turbulence. The relationship between vortex shedding and boundary layer evolution was a matter of interest since the early works of Roshko (1961) and Bearman (1969). As reported in the work of Roshko (1993) by analyzing near-wake hot-wire anemometry data and pressure distributions on the cylinder surface, the vanishing of the laminar separation bubble can be deemed as a mark of the onset of the critical regime. In Bearman (1969) spectral analysis of the wake signal suggests a strong link of the drag crisis to the disappearance of the narrow-band vortex shedding. In his work, oil-film was employed to qualitatively assess the state of the separation bubble upon tripping of the boundary layer. A step forward towards the understanding of the boundary layer evolution associated with the different flow regimes is taken by Achenbach (1968) and Achenbach (1975) where a systematic study of the skin friction distribution onto the cylinder surface is presented. The limitations of these works reside in the lack of temporal resolution and the point-wise character of the data. Furthermore, as stated by the authors, the intrinsic limitations of the measurement system hinder the reliability of the results as the boundary layer becomes turbulent. Recently, oil-film measurements by Miau et al. (2011) showed a marked three-dimensionality in the flow separation/reattachment behaviour and this was suggested to be associated with the unsteadiness of the pressure signal onto the cylinder surface although a direct correlation could not be established due to the average nature of the oil-film measurements. The relationship between the critical regime and the instability of the shear layer was directly investigated by means of large eddy simulations in the work of Singh and Mittal (2005) who concluded that the shear-layer vortices play a major role in boundary layer transition. The majority of these works have disclosed complex patterns of flow detachment and re-attachment characterizing the flow around a circular cylinder (Zdravkovich, 1997), with a strong dependence on the Reynolds number as well as by boundary conditions (aspect ratio, blocking factor, incoming turbulence level, surface roughness). As appears from the literature review, the relationship between the development of the boundary layer and the shear layers has been often subject of study and is not yet fully understood. To the authors' knowledge, the majority of works on the subject focuses on either near-wall or surface measurements, where the latter is usually represented by time-average, point-wise data or is subject to limitations as regards the turbulent boundary layer (Achenbach, 1968). These conditions hinder substantially the understanding of the complex interactions expected to take place between the boundary layer and the shear region.

By overcoming such limitations, in this work we present combined optical measurements by means of high-speed Particle Tracking Velocimetry (PTV) and high-speed temperature sensitive paint with the aim of investigating the flow as it undergoes transition between the transition-inshear-layer regime and the transition-in-boundary layer regime. In particular we aim at shedding light onto the link between the boundary layer development, the wall shear-stress topology, and shear layer evolution by means of time-resolved and whole-field observation of the surface temperature and relative skin friction and their instantaneous correlation to the near-wake velocity data.

The method of temperature sensitive paint, hereafter TSP, is based on temperature-sensitive coatings to obtain qualitative and quantitative evaluation of the thermal field on a model surface. TSP coatings, when excited by narrow-band, incident light, emit a temperature-dependent longer wavelength light (Stokes shifted) that can be detected by photosensitive devices (i.e., CCD/CMOS cameras). TSP has become a standard tool in transition measurements (Liu, 2004 and Yorita et al., 2012) especially in cryogenic wind tunnels (Fey et al., 2006) and whenever infra-red thermography cannot be employed, i.e., in water (Fey et al., 2013 and Capone et al., 2015). Recently Liu and Woodiga (2011) and Liu (2013) proposed a new method which can obtain relative skin friction field from single TSP images. 
Combination of PTV and TSP conveys the simultaneous and twofold perspective of both the phenomena occurring onto and close to the cylinder surface, via surface TSP data, and within the cylinder near-wake, via PTV. The high acquisition frequency makes it possible to study phenomena featuring complex and multi-scale dynamics, without the need for sensor installment onto or in proximity of the model surface. This represents a distinctive advantage whenever phenomena related to the boundary layer instability are to be observed and in circumstances where the flow is most sensitive to disturbances, as in the critical regime. The presented work aims at providing a novel insight into the relationship between boundary layer behaviour (laminar separation, circulation bubble, and turbulent reattachment), wall shear-stress topology, and shear layer dynamics at sub-critical and critical regimes, based on combined time-resolved results from near-wake velocity measurements, temperature and relative skin-friction surface data, and their correlation. In particular, the results obtained by whole-field surface measurements convey a perspective of the flow which is usually difficult to achieve due to the strong gradients occurring at specific conditions (e.g., close to flow separation or reattachment, Wu et al., 2000) and often requiring ad-hoc experimental techniques.

Section II provides a description of the apparatus and details about the experimental program, along with a description of the TSP and PTV methodology. The rationale for relative skin friction calculation is also described. In Section III results are presented and discussed. Mean values obtained from velocity data are shown and matched to temperature profiles and relative skin friction maps. Time-resolved and spectral data derived from TSP and PTV are reviewed and their correlation discussed.

\section{METHODS AND SETUP}

The facility consists of a closed-loop water tunnel having a 1:5.96 contraction nozzle and a square test section of side $B=600 \mathrm{~mm}$. Free-stream turbulence intensity and flow uniformity at the channel centerline are respectively $1.5 \%$ and $0.4 \%$. The test section is bounded by perspex windows in order to allow optical access from all directions. The hollow cylinder model made from aluminum, diameter $D=36 \mathrm{~mm}$ and $13.5 \mathrm{~mm}$ thickness, is mounted onto the tunnel perspex windows by means of two flanges. The cylinder is placed vertically and perpendicular to the main flow direction. Cylinder length $L=600 \mathrm{~mm}$ is equal to the test section width and consequently the aspect ratio is $L / D=16.7$ whereas the blockage factor is $D / B=0.06$. The experimental setup, depicted in Fig. 1, allows simultaneous optical acquisition of the flow thermal signature onto the cylinder surface via TSP combined with time-resolved measurement of the velocity field in the

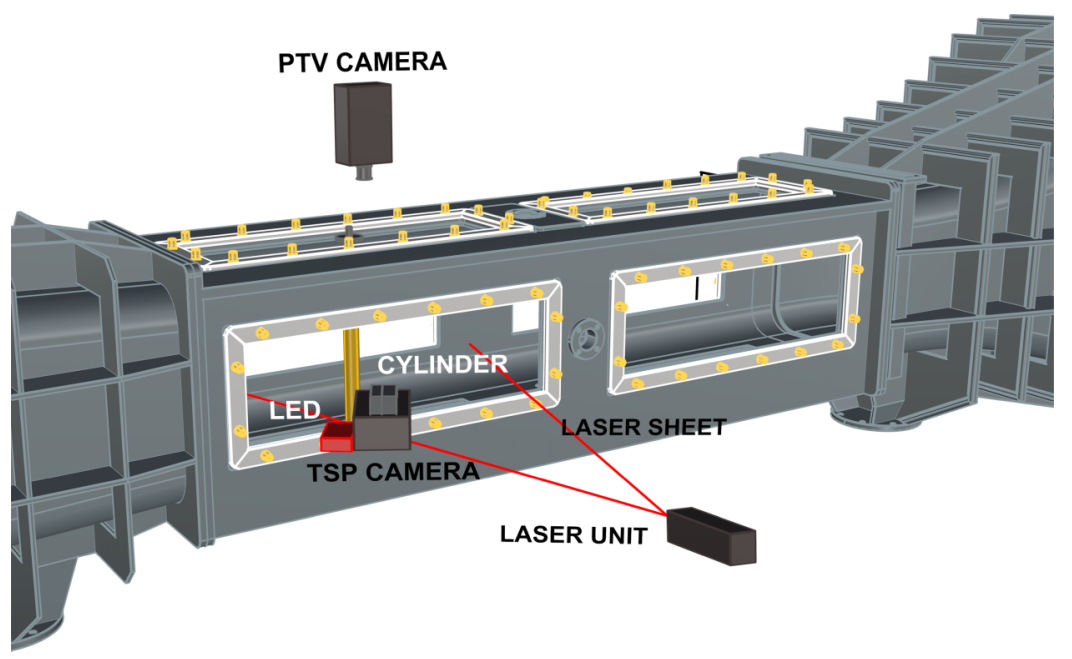

FIG. 1. CEIMM water tunnel experimental setup. Flow from right to left. 
near-wake by means of particle velocimetry. The experiments comprise five flow speeds, with free stream velocity $U_{0}$ ranging from $2.2 \mathrm{~m} / \mathrm{s}$ to $7.6 \mathrm{~m} / \mathrm{s}$. Corresponding Reynolds numbers, based on cylinder diameter and water kinematic viscosity at $25^{\circ} \mathrm{C}$, lie within the range $69000-238000$.

\section{A. Temperature sensitive paint}

Development and features of the TSP employed in this work have been described by Ondrus et al. (2015) and by Fey et al. (2013) and its suitability for water applications has been shown in the works of Capone et al. (2015) and Fey et al. (2013). Surface roughness and relative roughness of the TSP-coated cylinder are $R a=0.09 \mu \mathrm{m}$ and $R a / D=2.5 \times 10^{-6}$, respectively. In order to establish a thermal gradient on the cylinder surface for the TSP signature to be characterized by an approprate signal-to-noise ratio (Tropea et al., 2007 and Klein et al., 2015) the cylinder is warmed up by an internal flow of water supplied by an external thermostatic bath. In the given experimental conditions, the effect of natural convection has been shown to be negligible (Capone et al., 2015).

TSP acquisitions are carried out with a CMOS high-speed camera Photron Fastcam SA1.1, 1024 square pixels resolution, 12 bits dynamic range, which is placed tilted with respect to the flow (see Fig. 1) approximately $300 \mathrm{~mm}$ from the channel wall. The camera is equipped with Nikon $50 \mathrm{~mm}$ focal length lenses with 1.8 maximum aperture and a long-wave pass filter having a 50\% transmittance cutpoint at $600 \mathrm{~nm}$ wavelength. TSP layer excitation is provided by four high-power LEDs, nominal minimum and maximum peak wave length 400-405 nm, respectively. For all Reynolds numbers, a set of 10000 TSP images was collected with an acquisition rate of $1 \mathrm{kHz}$ covering an area of approximately $150 \times 150 \mathrm{~mm}^{2}$, corresponding to $4.1 \mathrm{D}$ and centered upon the mid-upper quarter of the cylinder. The cylinder surface angle covered by the TSP camera's field of view is from $\varphi_{1}=47^{\circ}$ to $\varphi_{2}=156^{\circ}$ with respect to the stagnation point, according to the polar reference system shown in Fig. 2.

Images are processed according to the procedure set out in Fey et al. (2013) and Capone et al. (2015). Dark images are acquired without excitation to account for the background noise. Reference images are acquired with LED lights on and without heating, under known temperature $T_{\text {ref }}$. Run images are obtained with heating on in order to enhance surface temperature difference, as explained previously. The average dark image is then subtracted from the average reference image and for each single run image, and a ratio is computed of the latter to the former. This procedure is effective in removing the disturbances due to residual ambient light, non-uniform illumination due to lighting and model curvature, uneven coating, and non-homogeneous luminophore concentration in the TSP active layer (Liu, 2004). With an appropriate calibration curve (not shown here), mean temperature on the cylinder surface can be extracted from TSP images (see Tropea et al., 2007 and Liu, 2004 for further details).

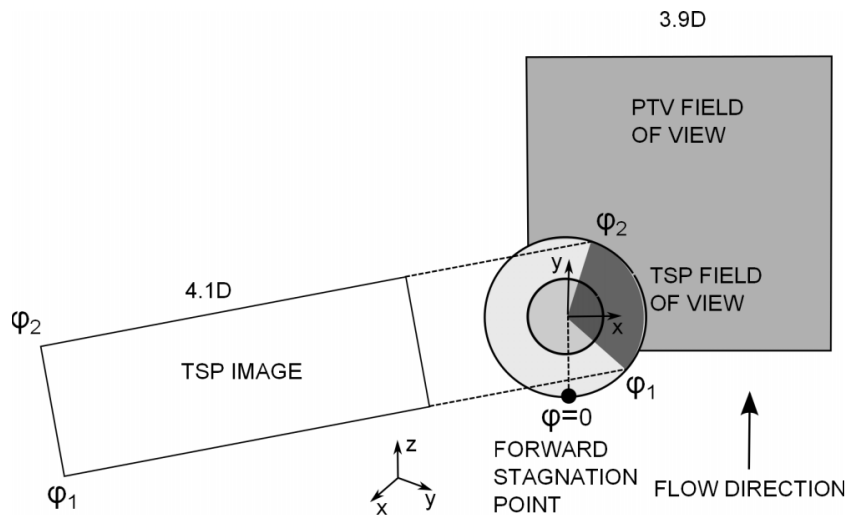

FIG. 2. Schematics of PTV and TSP acquisitions field of view. 


\section{B. Skin friction evaluation}

Insight into the evolution of the boundary layer state can be obtained via TSP measurement, due to the relationship between surface temperature gradients and the wall shear stress $\tau$ provided by the energy equation, (Liu and Woodiga, 2011),

$$
\rho c_{p} \frac{D T}{D t}+p \frac{\partial U_{j}}{\partial X_{j}}=k \nabla^{2} T+\mu \Phi
$$

projected on the wall surface.

Here $U_{j}(\mathrm{j}=1,2,3)$ are the velocity components, $p, \rho, c_{p}, k$, and $\mu$ are respectively the pressure, density, specific heat at constant pressure, thermal conductivity, and dynamic viscosity of the fluid, and $\Phi$ is the dissipation function. By means of Taylor expansions of the velocity components and temperature, in the limit $X_{3} \rightarrow 0$ of the coordinate normal to the surface (see Liu and Woodiga, 2011 and Liu, 2013 for the details), we obtain

$$
F+\tau_{i} \frac{\partial T_{w}}{\partial X_{i}}=0,(i=1,2),
$$

where $\tau_{i}$ are the components of the wall shear stress, $T_{w}$ is the surface temperature, and the quantity $F=F\left(\frac{\partial q_{w}}{\partial t}, \frac{\partial^{2} q_{w}}{\partial X_{i} X_{i}}, T, \Phi\right)$ is a function of the temporal and spatial gradients of the wall heat flux $q_{w}$ and of the thermal diffusion and dissipation. In a sense, Eq. (2) may be considered as a differential form of Reynolds analogy and its structural equivalence with the optical flow equation (Horn and Schunck, 1981) suggests the use of a variational approach to extract the relative skin friction as an inverse problem (Liu and Woodiga, 2011). From a physical perspective, Eq. (2) states that, in the absence of external phenomena, the temperature gradients are displaced by the skin friction. Since laminar boundary layer is associated with values of the wall shear stress that are lower than the turbulent state, it descends that also the heat transfer coefficients differ for each state, according to Eq. (2). As a direct result the turbulent boundary layer is associated with a lower surface temperature than the laminar state.

\section{Particle velocimetry setup}

A twin camera and lenses of the TSP setup are used to attain particle velocimetry measurements. A laser sheet, approximately $2 \mathrm{~mm}$ thick, is generated with a Spectra Physics Ar-ion laser, with a maximum power equal to $6 \mathrm{~W}$. The flow is seeded with neutrally buoyant $12 \mu \mathrm{m}$ diameter hollow glass spheres. 10000 images were stored for each Reynolds number, acquired at a rate from 2 to $6 \mathrm{kHz}$, depending on the flow speed. Each series contains a number of vortex shedding periods (i.e., wake oscillations) ranging approximately from 60 to 83 . The covered area, as shown in Fig. 2, is a 3.9D-sided square which extends up to 3.6D downstream of the cylinder and 3.2D in the spanwise direction.

Images were analyzed by means of a correlation-based PTV technique, derived from the locally solved optical flow equation (Lucas et al., 1981). The algorithm has been implemented in the software YATS (Miozzi, 2004), which contributed to the III (Stanislas et al., 2008) and IV International PIV Challenge (here as CNR-INSEAN team code). An interrogation window of 25 pixels weighted by a Gaussian filter centered on the interrogation window and having a standard deviation of 12 pixels has been considered. Images have been preliminarily conditioned by reiterating the application of a Wiener filter. Two equivalent datasets are obtained from the analysis, one containing the trajectory evolution of each particle and the other related to the time sequence of the snapshots of the sparse velocity fields extracted from each image pair. The former provides directly the Lagrangian description of the particle motion, whereas the latter is used to obtain the time-resolved velocity field description on a PIV-style Eulerian grid. All the data shown in this work are extracted in a Lagrangian fashion, i.e., the Eulerian flow field is evaluated from the instantaneous set of particle trajectories, which is represented by a sparse velocity vector field (Miozzi, 2005). The resolution of the resulting Eulerian projected grid is $0.75 \mathrm{~mm}$ per cell. 


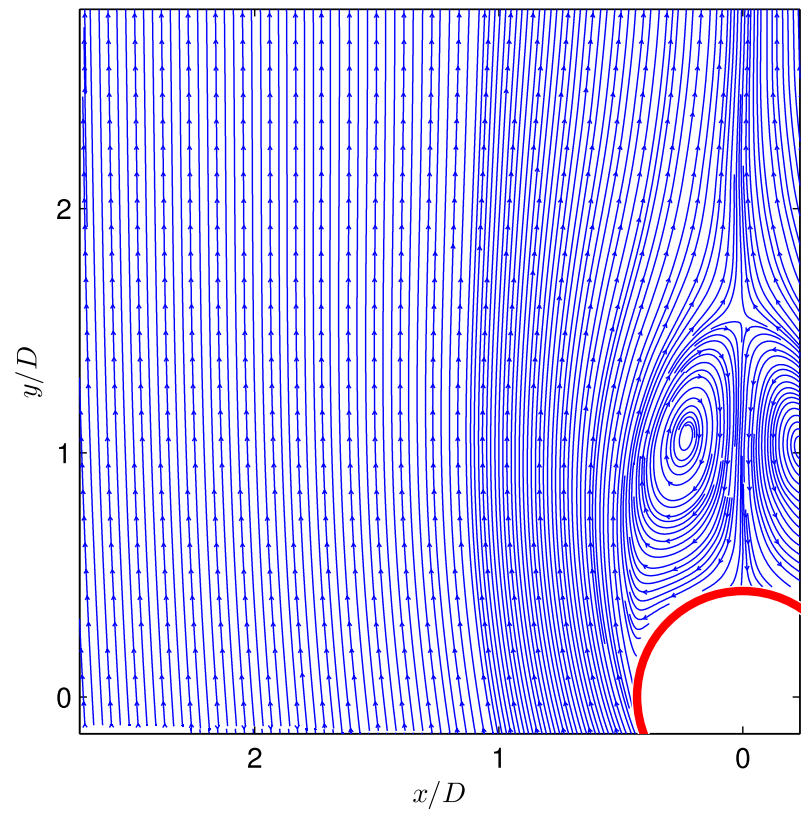

FIG. 3. Streamlines of the mean field at $R e=125000$.

\section{RESULTS}

\section{A. Mean values and topology}

The topology of the flow discussed in this section is based on the Reynolds averaging decomposition $U_{j}=\left\langle U_{j}\right\rangle+u_{j}$. In Fig. 3 the mean-flow streamlines are shown for $R e=125000$. As expected, two wide recirculation regions are noticeable due to the passage of the alternating vortices. The change of sign of the mean streamwise component of velocity along the rear axis defines the dimensionless recirculation length $l_{c}=1.45$. It depends strongly on the boundary conditions (aspect ratio, blocking factor, and turbulence intensity) and values ranging from 1.1-1.4 have been reported by several authors in this Reynolds number interval (Perrin et al., 2008; Cantwell and Coles, 1983; and Djeridi et al., 2003 among others). Consequently, the measured value appears reasonable. Simulations and experiments by Perrin et al. (2008) reported the occurrence of time windows of irregularity not associated with a specific frequency, for which the quasi-periodic component of the fluctuations is strongly attenuated. Analysis of velocity temporal sequences showed a similar behaviour also in the current experiment (not shown) and explains small asymmetries noticeable in Fig. 3. The uncertainties are estimated to be of the order of $2 \%$ and $3 \%$, respectively, for streamwise and spanwise mean components, with a 95\% confidence interval based on Student distribution. The normalized normal and mixed components of the Reynolds stress are shown in Fig. 4. The overall level of turbulence is comparable to the results from Cantwell and Coles (1983) and lower than the data reported in Perrin et al. (2007), obtained at a comparable Reynolds number. The streamwise component $\left\langle u^{2}\right\rangle$ features the typical two-lobe structure with a maximum located nearly at $y / D=1.1$ and $x / D=0.5$ whereas on the rear-axis the maximum value is reached at approximately $y / D=1.55$. The single-lobe shape of $\left\langle v^{2}\right\rangle$ has its peak, equal to 0.25 , on the rear axis at $y / D=1.6$. The mixed Reynolds stress reaches its maximum far from the axis, at $x / D=0.3$ and $y / D=1.5$. The locations of the maxima are consistent with the results from Singh and Mittal (2005) and Perrin et al. (2007) $(L / D=4.8, D / B=0.21)$ considering the strong variability of the flow features with boundary conditions especially near the critical regime.

In Fig. 5 the turbulent kinetic energy is presented for the investigated Reynolds numbers, normalized to the free stream velocity $U_{0}$ at each speed. The results show a substantial invariance of the spatial pattern up to $R e=170000$ where an overall decrease in relative turbulence level occurs. At $R e=238000$ a marked difference is reported in the structure of the shear layers which adhere 


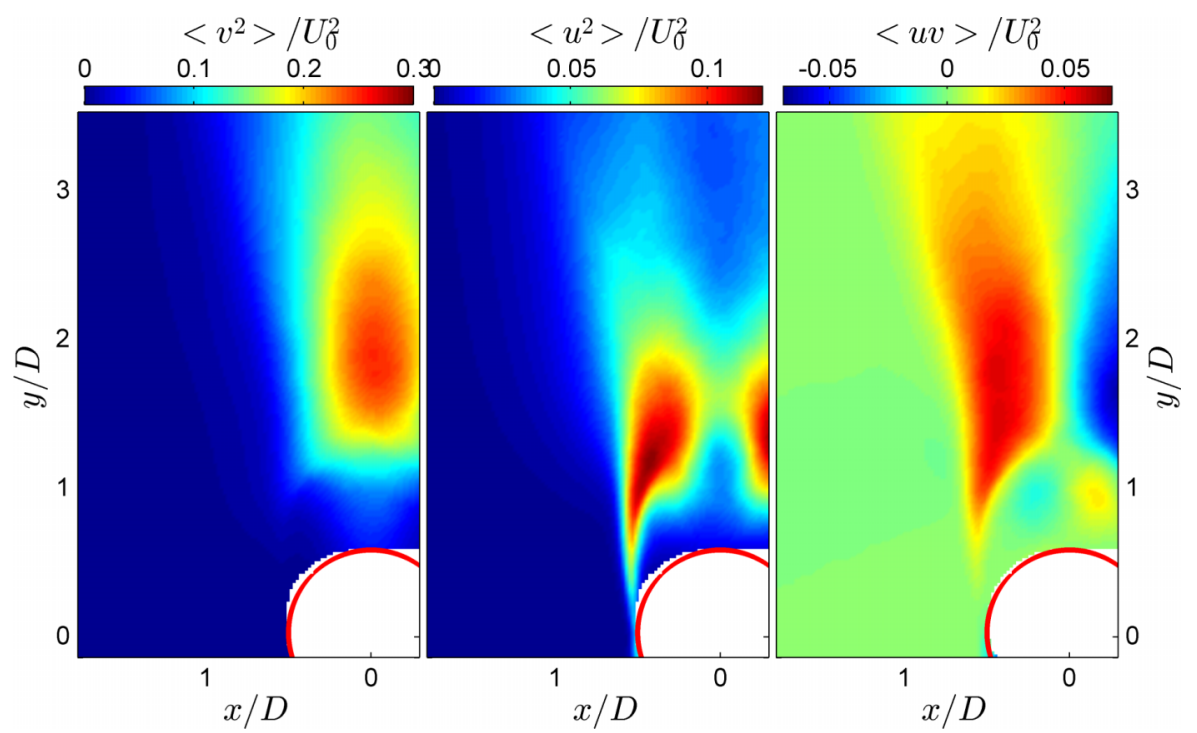

FIG. 4. The normal and mixed Reynolds stress components are plotted at $R e=125000$.

more to the cylinder surface consequently moving to each other. This behaviour, associated with the reduction of the near-wake width, has been described by Zdravkovich (1997) and reported in simulations by Singh and Mittal (2005) and is considered strictly linked to the transition to turbulence undergone by the boundary layer and the occurrence of its reattachment onto the cylinder surface. Fig. 6 shows the mean temperature field on a cylinder section approximately $1.4 \mathrm{D}$ long, obtained at $R e=69000$. Several qualitative and quantitative information can be inferred from the complex thermal trace visible on the surface and a comprehensive study of this subject is provided in Capone et al. (2015). For the purpose of the current work, we focus on the dark (i.e., warmer) straight region visible at approximately $\varphi=79^{\circ}$ for $R e=69000$. This straight line is associated with the occurrence of flow separation, which causes a reduction in the wall shear stress and a consequent higher surface temperature as per Eq. (2). We build on this observation to plot the separation angle $\varphi_{s 1}$ versus modified Reynolds number as shown in Fig. 7 where error bars are defined according to the image intensity variance during acquisitions (see Capone et al., 2015 for further details). The sensitivity of the critical Reynolds number to boundary conditions, in particular to free-stream turbulence, has been highlighted by many authors and has been the focus of several studies (Norberg and Sunden, 1987; Kiya et al., 1982; Sadeh and Saharon, 1982; and Kwok, 1986 among others). Zdravkovich (1997) points out that the free-stream turbulence acts as a random excitation of free-shear and boundary layers. As the flow approaches the transition-in-boundary layer state the boundary layer becomes unstable and the effect of free-stream turbulence is significant. Kwok (1986) reports that small-scale turbulence near the stagnation line promotes boundary layer early transition and delay in separation, thus effectively raising the Reynolds number. Sadeh and Saharon (1982) reach the same conclusion highlighting that the modifications undergone by the boundary layer are in agreement with the model proposed by the vortex-amplification theory. An effort to correlate the free-stream turbulence characteristics to an effective Reynolds number is made by Kiya et al. (1982). They propose the relationship $R e_{c}^{1.34} T a=K$, where $R e_{c}$ is the critical Reynolds number for which the drag coefficient is equal to $0.8, K=198000$, and $T a$ is the Taylor number. The latter accounts for the intensity and scale of the free-stream turbulence and is expressed as $T a=\sqrt{\left\langle u^{2}\right\rangle+\left\langle v^{2}\right\rangle} / U_{0} /\left(D / L_{x}\right)$ in the modified form proposed by Arie et al. (1981) with $L_{x}$ representing the integral scale of streamwise turbulence. The plot of the separation angle $\varphi_{s 1}$ versus the modified Reynolds number $R e / R e_{c}$ in Fig. 7 shows a good match to the literature data with a steep rise in $\varphi_{s 1}$ as the critical Reynolds number is reached, which is further evidence of the boundary layer transition to turbulence. 


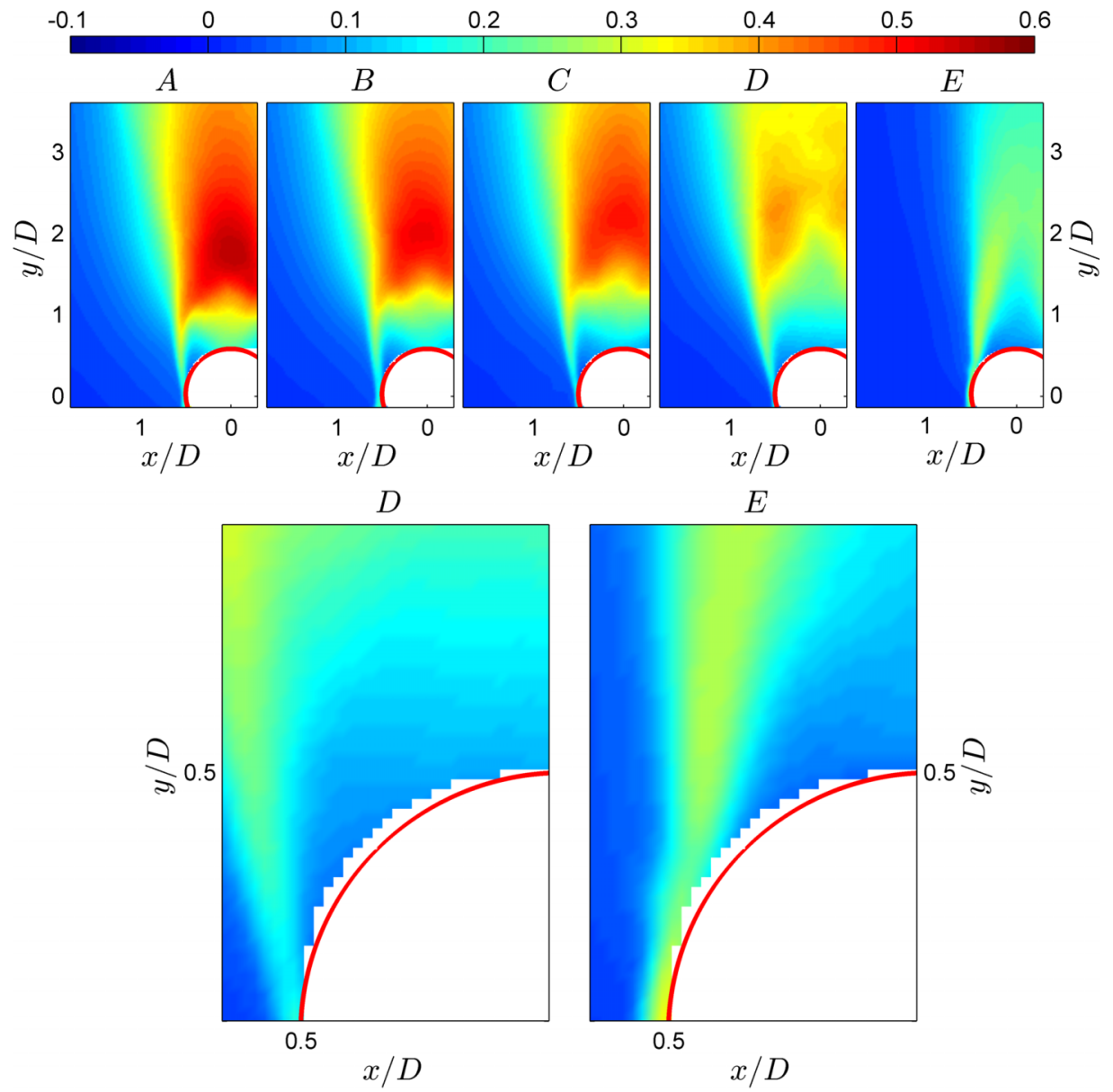

FIG. 5. The normalized turbulent kinetic energy is plotted for Reynolds number varying from 69000 to 238000 . From left to right (a) $R e=69000$, (b) $R e=97000$, (c) $R e=125000$, (d) $R e=170000$, and (e) $R e=238000$. For highest Reynolds numbers (case (d) and (e)), magnification of the region close to the cylinder surface is shown.

Mean temperature profiles on the cylinder surface are compared in Fig. 8. Overview of the results shows that the overall mean temperature onto the cylinder surface decreases, as expected, due to increasing heat transfer as the flow free stream velocity increases. Furthermore, two distinct regimes can be identified: for $R e<=170000$ the initial temperature rise due to the oncoming laminar separation is followed by a mild descent. The peak in temperature, corresponding to the separation point, shifts upstream matching the results reported in Fig. 7. At the highest Reynolds number the abrupt leap downstream of the laminar separation is followed by a steeper decrease of temperature than that observed at lower Re, which is associated with the transition to turbulence of boundary layer and the consequent rise in the heat transfer at the surface. A final increase in temperature is then registered at approximately $\varphi=140^{\circ}$, presumably due to the final flow separation.

High-resolution relative skin friction maps obtained from TSP are used to investigate the topology of the skin friction vector field onto the surface. Following the methodology described in Liu and Woodiga (2011) and Liu (2013), the relative skin friction vector fields from mean surface temperature maps are calculated. The variational method has been used to find the inverse solution to a Horn-Schuck optical flow (Eq. (2)) where the optical flow is replaced by the relative skin friction and the time derivative of image intensity by a source term $F$ (Liu and Woodiga, 2011). The perspective conveyed by skin friction measurements is meant to be complementary to the simultaneous velocimetry data in the near-wake. To this aim, mean relative skin friction maps are matched to flow streamlines and Reynolds mixed stress data in Figs. 9 and 10, respectively, at $R e=69000$ and $R e=238000$. The skin friction maps exhibit two kinds of features, which can be classified 


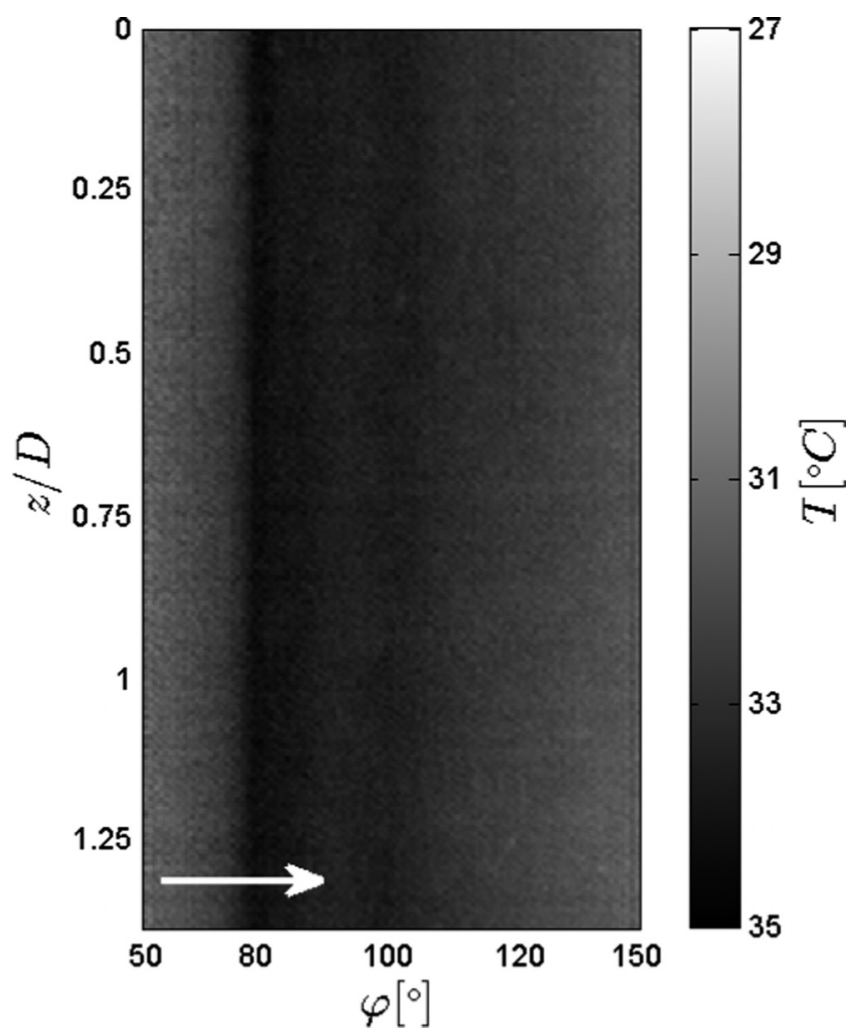

FIG. 6. Average temperature field on cylinder surface at $R e=69000$, obtained from TSP image. White arrow indicates flow direction.

as loci of convergence and divergence of skin friction streamlines. The spatial evolution of those features identifies the separation and reattachment lines, respectively (Wu et al., 2000).

The maps refer to a cylinder region approximately 1.4D long, immediately below the plane where velocimetry measurements are carried out. The first laminar separation point is clearly visible at $R e=69000$ as the first line of convergence encountered following the flow path around the cylinder and located approximately at $\varphi_{s 1}=78^{\circ}$. This region is followed by a second frontline of packed

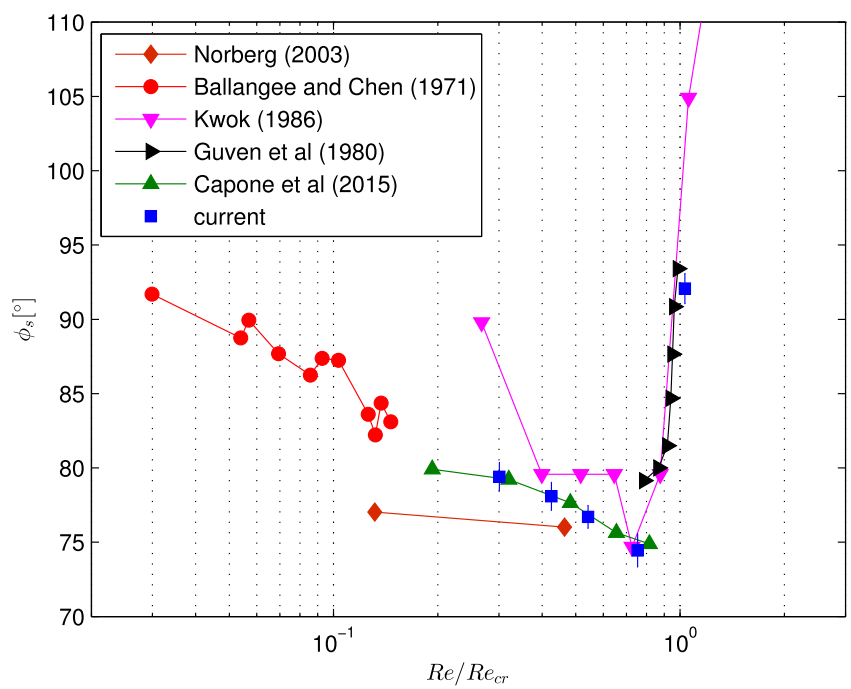

FIG. 7. The distribution of the mean separation angle is plotted as a function of the ratio $R e / R e_{C}$ for the current experiment and previous ones. 


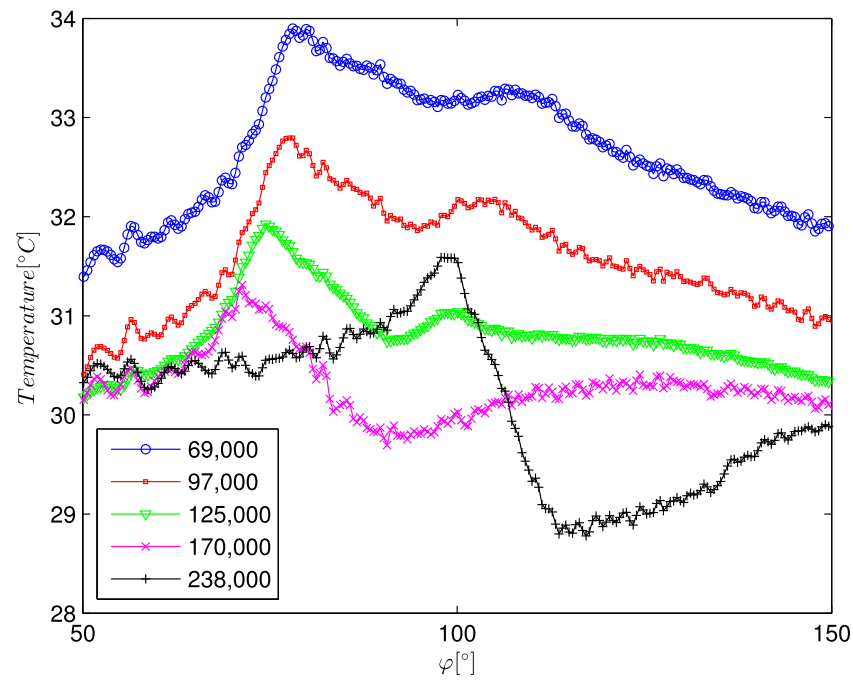

FIG. 8. The distribution of the mean temperature on cylinder surface as a function of the angle position for Reynolds numbers varying from 69000 to 238000 .

separation and reattachment regions for $95^{\circ}<\varphi<104^{\circ}$. These structures are typically unstable and are consistent with a scenario where the flow is undergoing intermittent reattachment, but the boundary layer is not yet sufficiently energetic to steadily re-attach onto the surface. Comparison to the mixed turbulent stress shows that the shear layers develop relatively far from the cylinder surface and after laminar separation. It descends that, in these circumstances, the separated laminar boundary layer does not experience, downstream of its separation, enough mixing by the action of the eddies generated by the shear layer vorticity (Singh and Mittal, 2005).

This condition persists virtually unchanged as the Reynolds number increases (not shown) up to $R e=238000$ when dramatic modifications take place. At this stage the boundary layer has sufficient energy to undergo turbulent re-attachment as suggested by the skin friction lines in Fig. 10. The closely packed region which characterizes the lower Reynolds number cases vanishes and the topology at the surface changes radically. A recirculation area, the so-called recirculation bubble, is thus established between the first laminar separation line and the secondary reattachment line with the laminar separation angle increasing up to $\varphi_{s 1}=95^{\circ}$ as expected for the one-bubble regime (Zdravkovich, 1997) and as already shown in Fig. 5. The eventual flow separation takes place
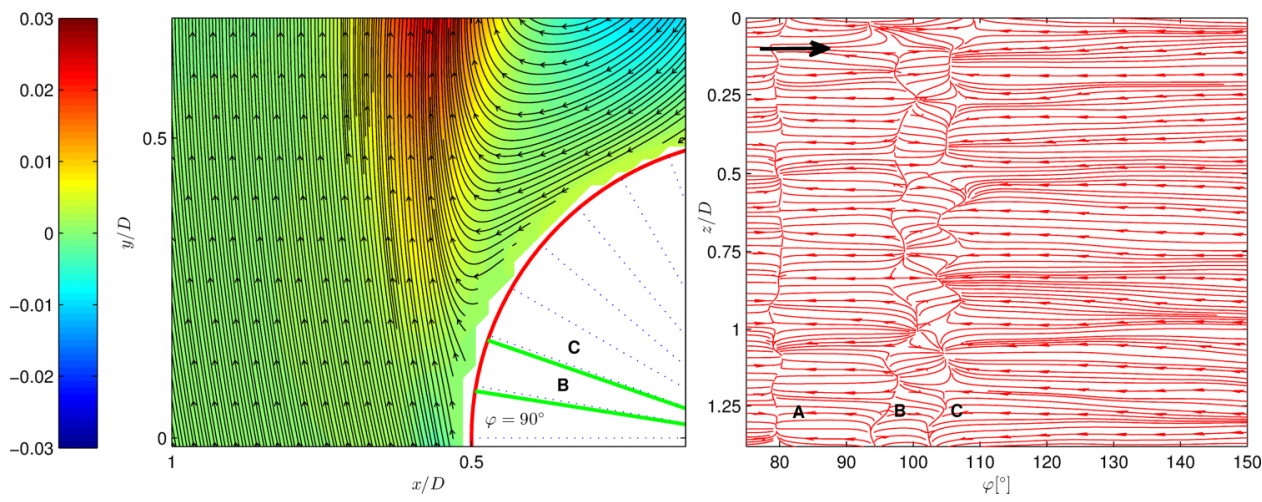

FIG. 9. Mean streamlines from PTV data overlayed onto mixed Reynolds stress on the background (left). Mean relative skin friction streamlines calculated from TSP images (right). Both data sets are obtained at $R e=69000$. Topological lines of skin friction convergence/divergence are highlighted and matched to the corresponding position on the left-hand figure: (a) convergence line associated with laminar separation (b) divergence line associated with reattachment (c) convergence line associated with final detachment. 

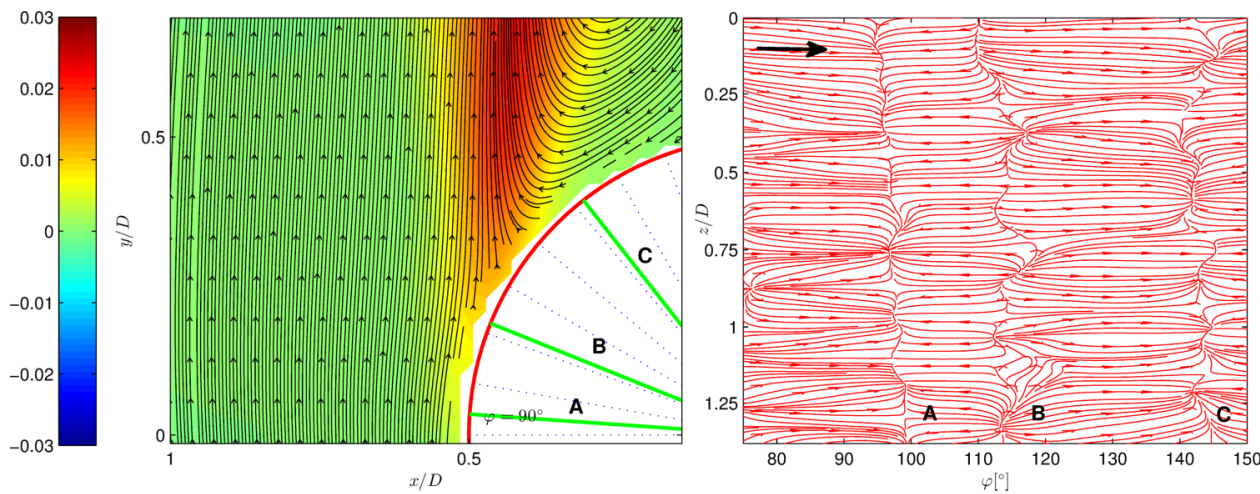

FIG. 10. Mean streamlines from PTV data overlayed onto mixed Reynolds stress on the background (left). Mean relative skin friction streamlines calculated from TSP images (right). Both data sets are obtained at $R e=238000$. Topological lines of skin friction convergence/divergence as defined in Fig. 9.

much further downstream accounting for the flow structure modifications leading to the drag crisis phenomenon and is marked by a third line of skin friction convergence at approximately $\varphi_{s 2}=140^{\circ}$. This scenario is confirmed by the mixed turbulent stress results displayed in Fig. 10, which show how at critical Reynolds number the shear layer instability grows immediately after laminar separation, as found by Singh and Mittal (2005). Close to the surface, the increase in turbulent stress is confined to the region corresponding to $100^{\circ}<\varphi<130^{\circ}$ with, as shown in Fig. 5, the shear layers closing up to each other. This range of angles compares well with the abrupt temperature drop shown in Fig. 8 and is consistent with a boundary layer becoming turbulent. Shortly afterwards, as conveyed by skin friction measurements, the shear layers develop far from the surface and, consistently, the turbulent boundary layer separates. Mean streamlines, from which the location of the final separation can be identified, confirm these findings, although at the highest Reynolds number the match to the skin friction results features lower quality, due to the strong velocity gradients close to the wall affecting velocimetry measurements.

Relative skin friction results are summarized in Fig. 11 where the angles of skin friction lines convergence/divergence are plotted versus Reynolds number. The laminar separation angle $\varphi_{s 1}$, represented by convergence line $\mathrm{A}$, and the reattachment angle, associated with the divergence line B, feature a similar behaviour as discussed for Fig. 7. The angle of last convergence line C

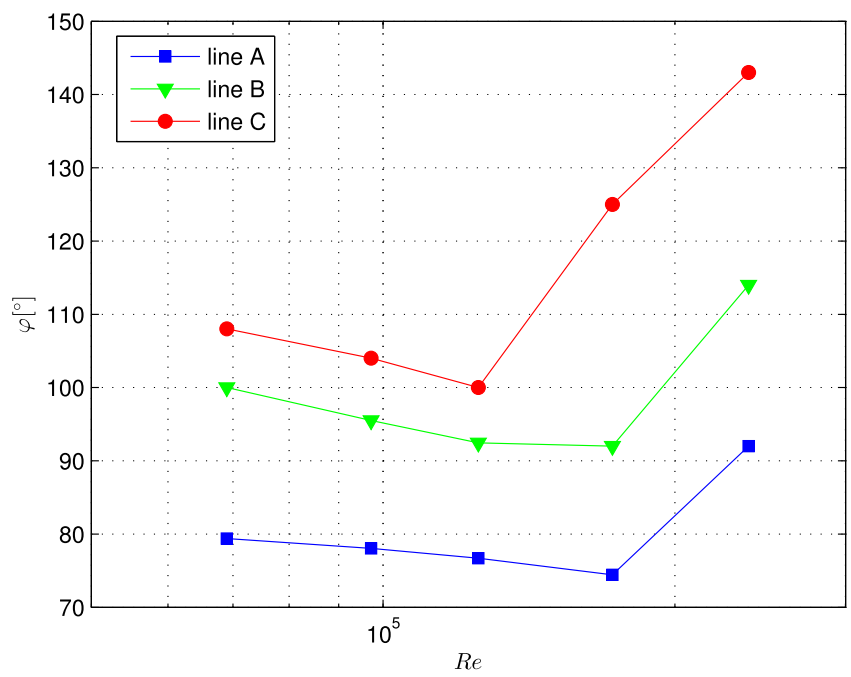

FIG. 11. The distribution of the angles of topological convergence/divergence of skin friction lines are plotted versus Reynolds number. Labels a, b, and c are referred to the topological locations shown in Figs. 9 and 10. 
undergoes a steep rise which anticipates the climb shown by $\varphi_{s 1}$ for $\operatorname{Re}=238000$. This behaviour, which is also visible in Fig. 8 as a lack of a sharp secondary peak in temperature, suggests that for $R e=170000$, the flow lies in an intermediate condition. This is associated with shear layers which are still developing far from the surface, as shown in Fig. 5, whereas the line of skin friction convergence associated with the final detachment is located at $\varphi$ angles characteristic of the critical regime.

\section{B. Instantaneous motion and spectral analysis}

In Figs. 12 and 13, a sequence of instantaneous vorticity fields, covering a time span of approximately one shedding cycle, is shown for $R e=69000$ and $R e=238000$, respectively. The vortex shedding is clearly visible at $R e=69000$ with smaller vortices entangled around the main vortical structure and detaching from it, as reported by Perrin et al. (2008) for similar Reynolds number.

The vortex shedding pattern remains substantially unchanged (not shown for the sake of brevity) up to $R e=238000$ where, as seen from Fig. 13, the near-wake becomes more disorganized due to the boundary layer turbulent transition and the closing up of the shear layers. Shedding is still noticeable, though it features a less regular behaviour with respect to lower Reynolds numbers, as reported by Bearman (1969). The normalized power spectra obtained from the time signal of the velocity streamwise component inside the shear layer $(x / D=0.5, y / D=1)$ are shown in Fig. 14 for all the Reynolds numbers tested. The energy peak associated with the shedding is clearly identifiable for $R e<170000$ and features a level which, compared to the background energy, remains quite constant as the speed increases. Second harmonics are also visible, which are progressively more energetic as the Reynolds number increases. At $R e=238000$, the reported abrupt change in the near-wake structure and the more irregular shedding are confirmed by the broadened energy peak, as found by Bearman (1969), and by a more homogeneous distribution of energy across the spectrum, which is a sign of a narrow wake. The shedding peak sharpness, defined as the ratio of the
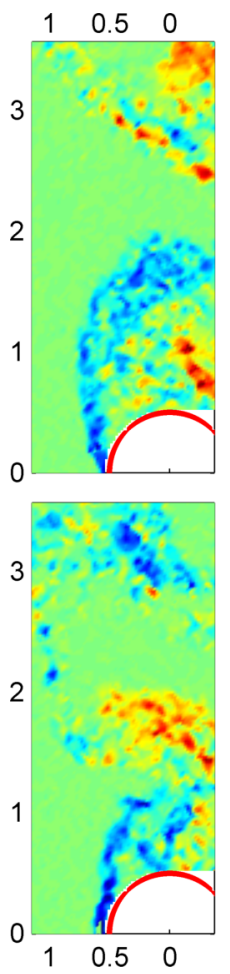
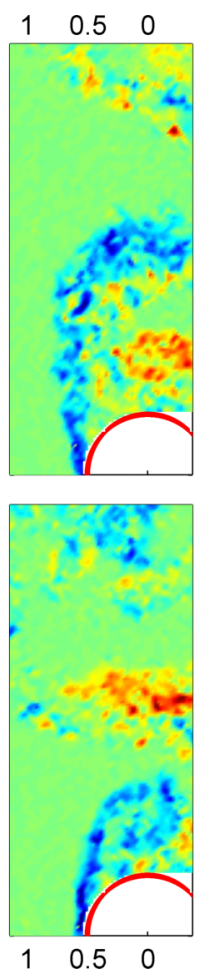
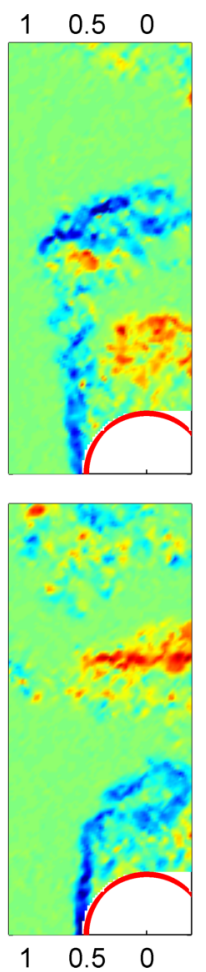
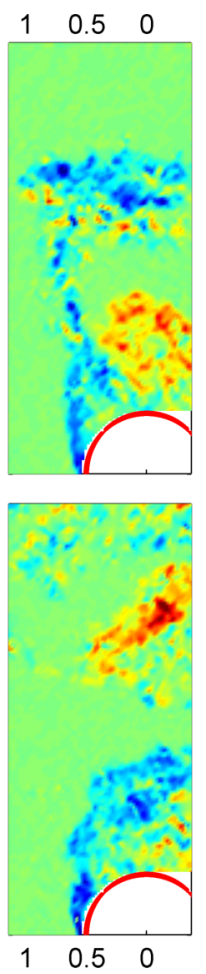
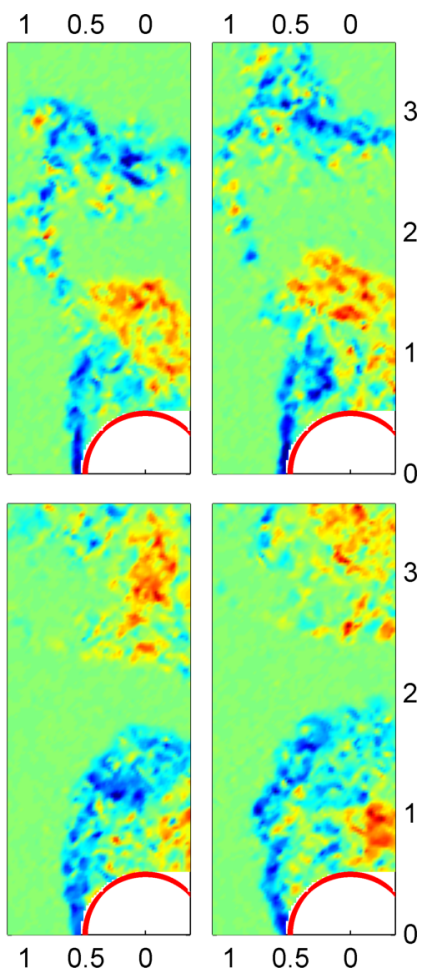

FIG. 12. A sample set of instantaneous vorticity fields is shown at $R e=69000$. The time step between each frame is $T=7.5 \mathrm{~ms}$. 

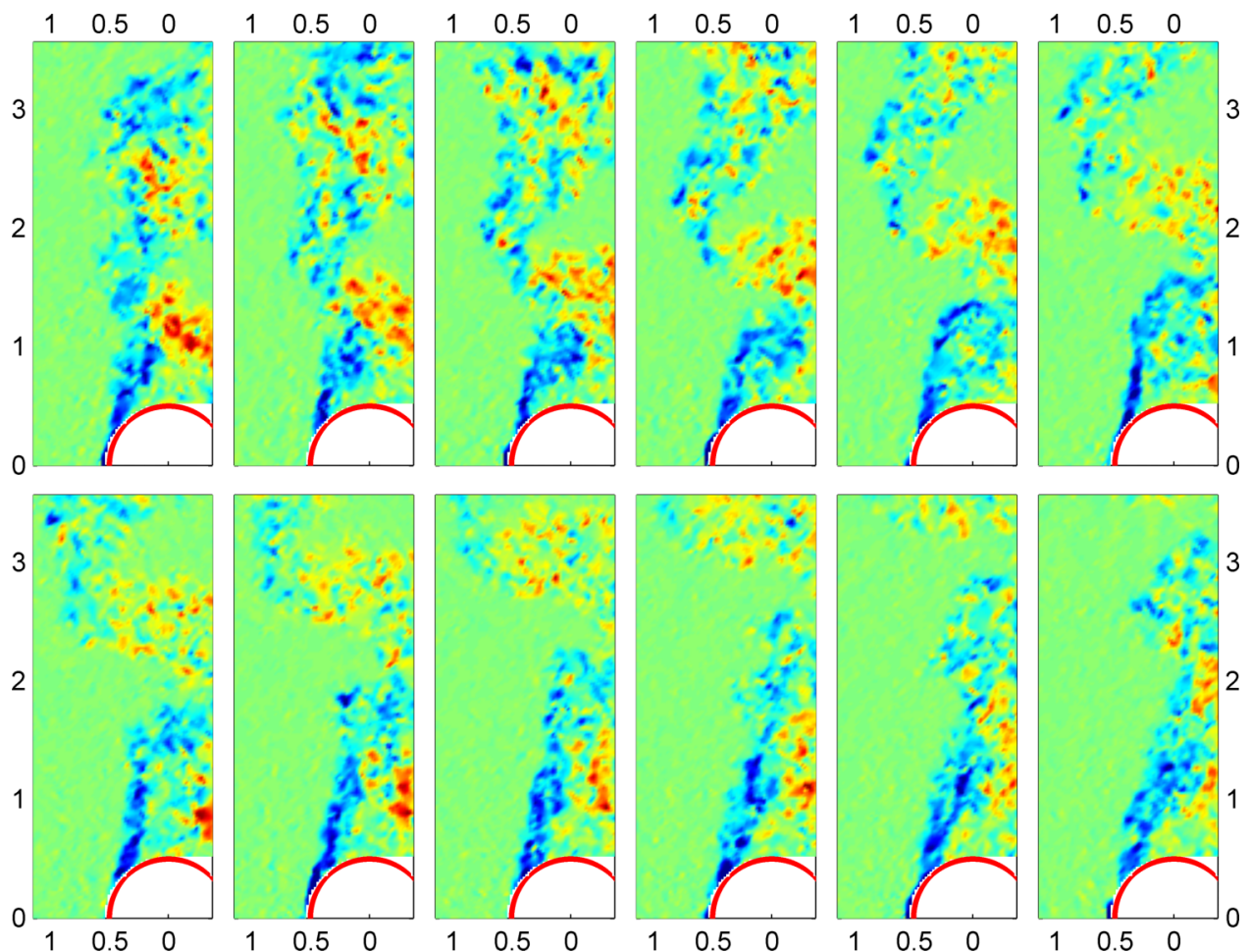

FIG. 13. A sample set of instantaneous vorticity fields is shown at $R e=238000$. The time step between each frame is $T=1.9 \mathrm{~ms}$.

peak frequency to the half-power bandwidth represents an index of the shedding regularity and is found to decrease from a value of $12.5-20$ for $R e<170000$ down to 6.25 at $R e=238000$.

In Fig. 15 we present two instantaneous TSP images obtained respectively at $R e=69000$ and $R e=238000$, conveying the strong modifications undergone by the surface thermal patterns at different flow regimes. Without going into detail of the fine structures (see Capone et al., 2015), at $R e=69000$, the thermal signature of the coherent vortical structures of the wake acting onto the surface can be noticed for $\varphi>80^{\circ}$. The extent of this region is remarkably reduced at the highest

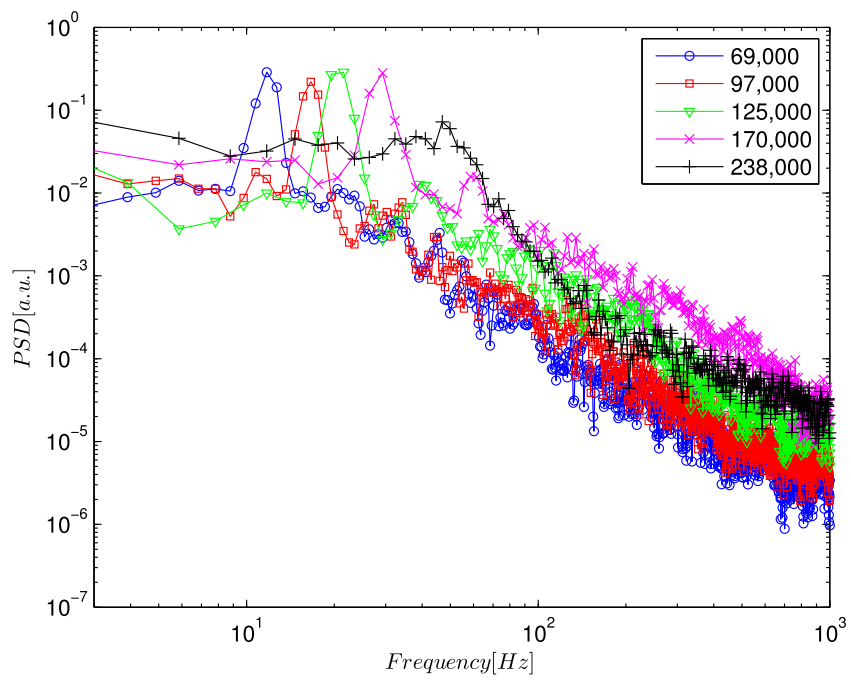

FIG. 14. The power spectral density obtained from the time history of the streamwise velocity inside the shear layer $(x / D=0.5, y / D=1)$ is plotted for Reynolds number varying from 69000 to 238000 . 

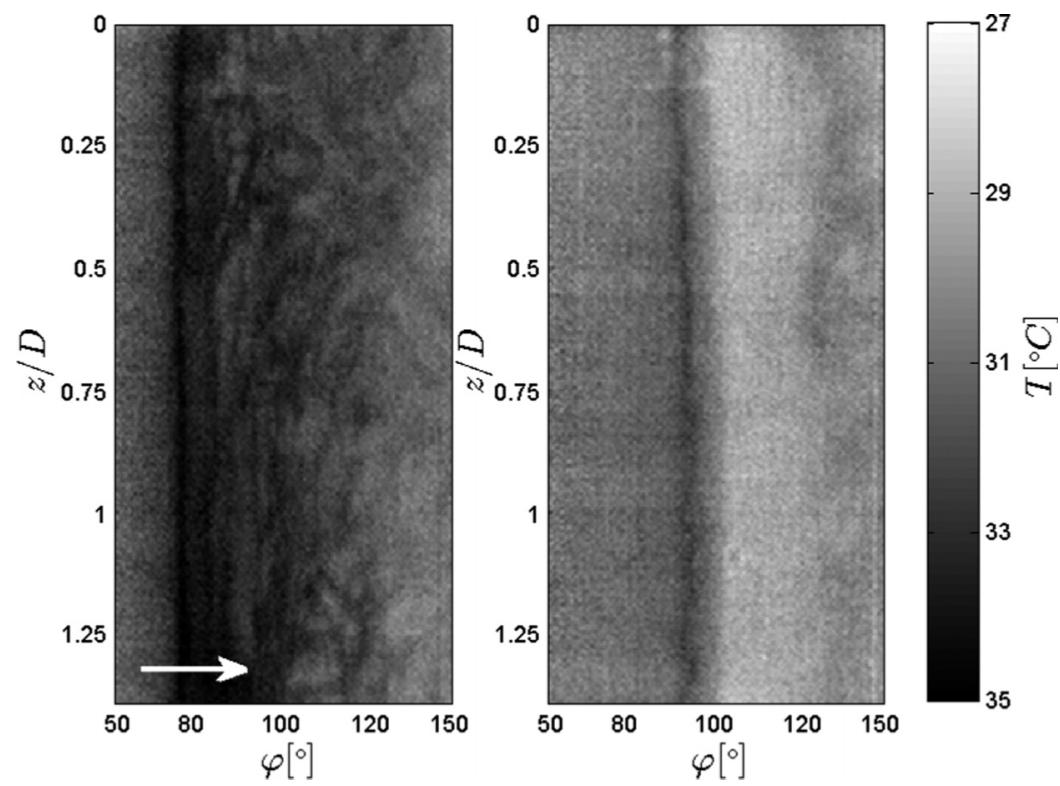

FIG. 15. Instantaneous temperature fields onto the cylinder surface obtained from TSP images are shown at $R e=69000$, left and $R e=238000$, right. White arrow indicates flow direction.

Reynolds number. At this flow speed, the signature is detectable as the darker area at approximately $\varphi>120^{\circ}$ and is characterized by weaker thermal gradients due to the overall higher heat transfer.

As carried out for the mean TSP images, the instantaneous separation angle is identified and its link to the shedding phenomenon is investigated. Fig. 16 shows the temporal evolution of the separation angle position along the cylinder span at $R e=125000$ obtained from instantaneous TSP images together with a sample time evolution at a specified location. The oscillating pattern is clearly visible, with the occurrence of bursts of irregular shedding, whose existence is reported also by experiments and simulation in Perrin et al. (2008).

Singh and Mittal (2005) suggested that the boundary layer turbulent transition is triggered by the shear layer instability. In agreement to this scenario we showed in Fig. 9 that at sub-critical Reynolds numbers, the shear layer instability develops only after laminar flow separation. Furthermore, the angle at which laminar flow detachment occurs is characterized by an oscillation of the same nature. This is confirmed by the normalized power spectral density of the separation angle temporal signal, reported in Fig. 17, which displays peaks located at frequencies matching the wake velocity signal. At $R e=170000$, the peak intensity drops (as shown in Capone et al., 2015) and, remarkably enough, at $R e=238000$, it vanishes completely. From this evidence, it is suggested that as the flow approaches and eventually enters the critical regime, the fluctuations in the near-wake

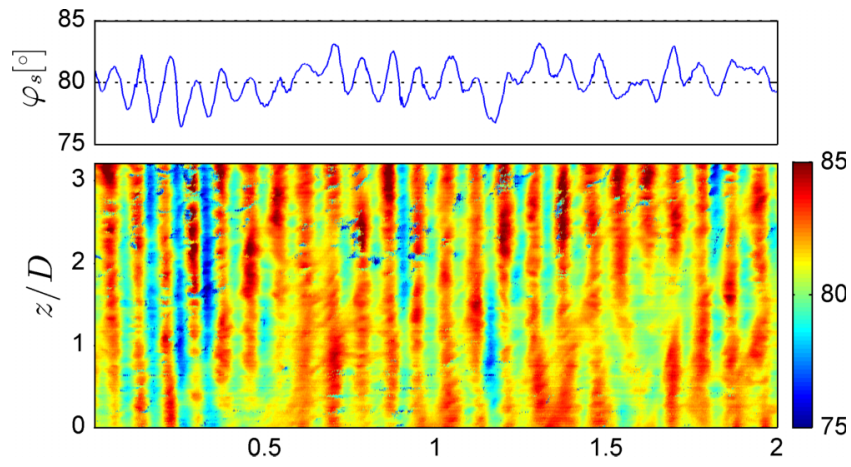

FIG. 16. Temporal evolution of the separation angle $\varphi_{s}$ at $Z=0.5 D$ and $R e=125000$, top. The temporal evolution of $\varphi_{s}$ is plotted for $0<Z / D<3$, bottom. 


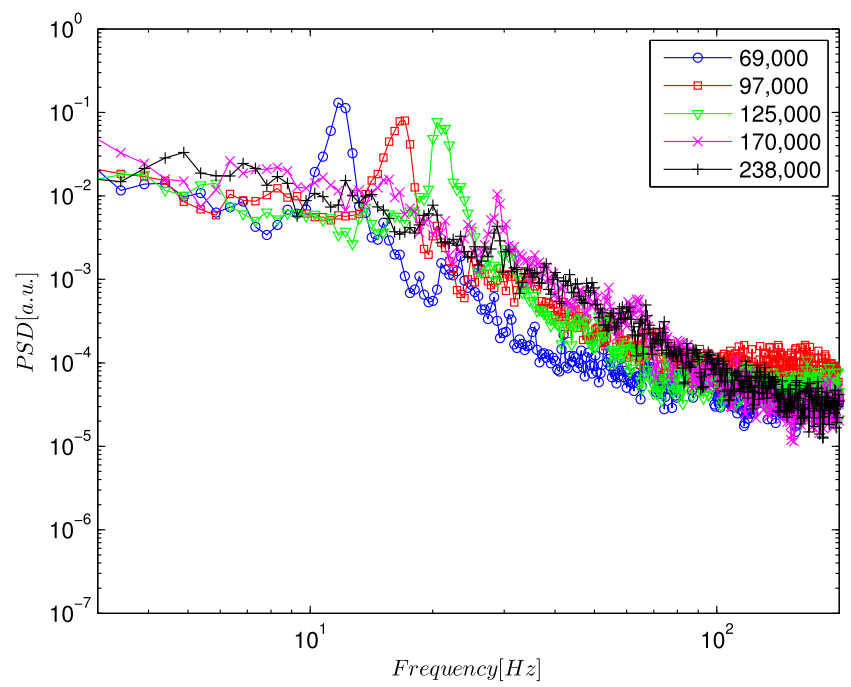

FIG. 17. The power spectral density obtained from the time history of the separation angle $\varphi_{s}$ is plotted for Reynolds number varying from 69000 to 238000 .

display a different structure from those of the separation point onto the cylinder surface. Further insight into this subject is provided by the correlation coefficient $\rho_{\text {corr }}$ of the time history of the separation angle $\varphi_{s}(t)$ and the local vorticity $\omega(t)$ calculated as

$$
\rho_{\text {corr }}=\left|\frac{1}{N-1} \sum_{i=1}^{N}\left(\frac{\varphi_{s}\left(t_{i}\right)-\overline{\varphi_{s}(t)}}{R M S\left(\varphi_{s}(t)\right)}\right)\left(\frac{\omega\left(t_{i}\right)-\overline{\omega(t)}}{R M S(\omega(t))}\right)\right| .
$$

Maps of $\rho_{\text {corr }}$ are shown in Fig. 18 for $R e=125000$ and $R e=238000$ with overlayed the contour of mean vorticity isolines. The strong correlation of the separation line oscillations and the vorticity fluctuations confirms the relationship between shear layer instability and laminar flow separation dynamics, in particular in the sub-critical regime. At critical Reynolds number, it appears that modifications in the relationship between separation oscillations and vortex shedding take place as pointed by spectral data analysis. As the flow enters this regime an overall drop of the correlation coefficient value is reported although a region close to the cylinder surface still presents a relatively higher-correlation two-lobe pattern. The reach of this region inside the near-wake is somewhat reduced compared to the sub-critical state. This occurrence is confirmed by the vorticity isolines distribution. These are characterized by a good match to the correlation maps for $R e=125000$ up to $\mathrm{y} / \mathrm{D}=2$, whereas at $R e=238000$ they feature a marked misalignment yet from approximately $\mathrm{y} / \mathrm{D}=0.5$, giving evidence of increasing independence between separation line oscillation and vortex shedding. Eddies generated by the shear layers reach upstream causing the laminar boundary layer to be more energetic and eventually to reattach and the separated boundary layer to behave no more as a free shear layer (Singh and Mittal, 2005). The turbulent boundary layer, whose thermal trace is visible as the brighter area visible downstream of the laminar separation in the instantaneous image in Fig. 15 is, due to its increased energy, less sensitive to the shedding in the near-wake and consequently increasingly uncorrelated. Consistently to this scenario, the power spectrum of the oscillations does not display a distinctive peak. This subtle difference is noticeable when comparing Figs. 12 and 13: in the first case the shear layers move in a swaying fashion and more importantly the same oscillation is observed close to the cylinder surface. In the second case, the shear layers are constrained by the flow structure modifications at high Reynolds numbers to move within a narrower region and the vorticity region close to the surface does not appear to follow the oscillation pattern. It was suggested by Bearman (1969) that regular vortex shedding be hindered and eventually stop because of the disruption of the recirculation bubble by the turbulent wedges coming from the front of the cylinder fostered by the growing three-dimensionality of the flow. The thermal trace of these impinging structures is visible in Fig. 15 for $125^{\circ}<\varphi<150^{\circ}$ and resembles the stronger 


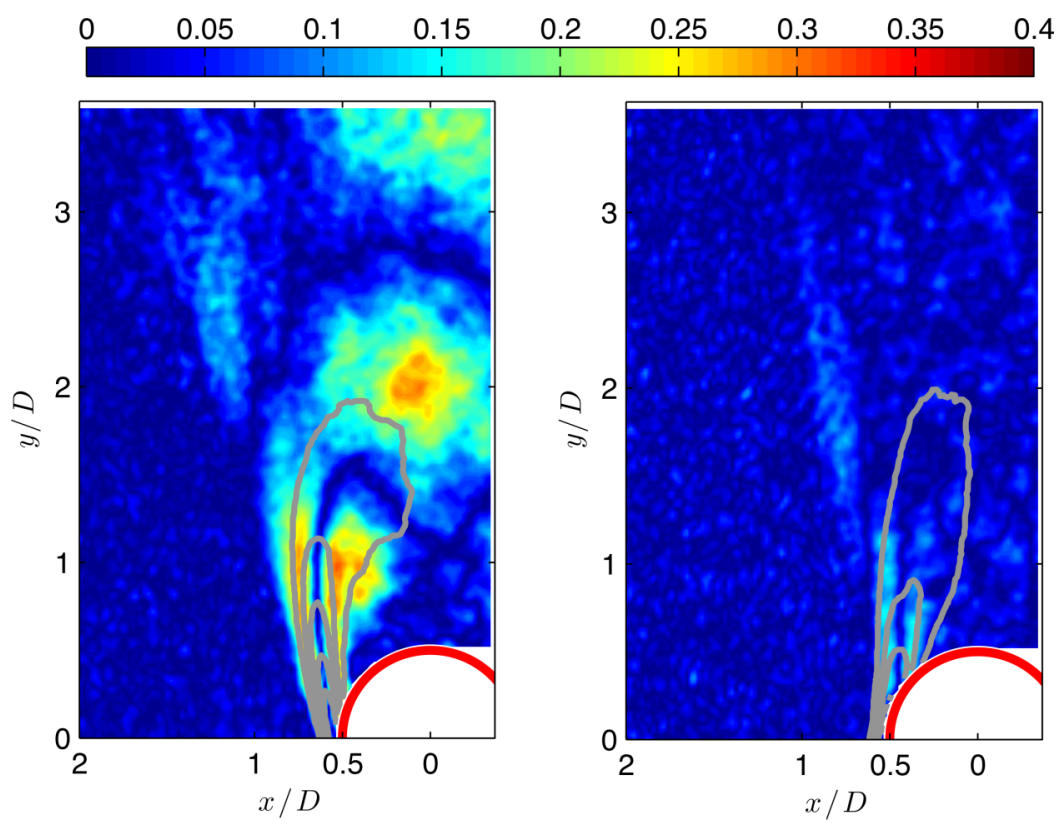

FIG. 18. Maps of correlation coefficient of separation angle temporal sequence $\varphi_{s}(t)$ and local vorticity $\omega(t)$ (color) and mean vorticity isolines (grey) at $R e=125000$ and $R e=238000$.

signature visible immediately downstream of the separation point for $R e=69000$. Observation of the time evolution of the separation line at different Reynolds numbers (not shown here) confirms that in spite of an overall consistency in the line straightness at sub-critical Reynolds numbers, the separation line features generalized bending at $R e=238000$, supposedly due to the increased threedimensional character of the flow.

From spectral data obtained via PTV and TSP in Figs. 14 and 17, we calculate the Strouhal number $S t=f * D / U_{0}$ of the vortex shedding and we compare it to the literature data in Fig. 19 . The substantial invariance of the Strouhal number in sub-critical regime is explained by the concurrent action of the shear layers closing up to each other and the increase in the recirculation length (Zdravkovich, 1997). At $R e=238000$ the shear layers tendency towards closing up to each other is not sufficiently countered and consequently the Strouhal number undergoes a steep increase

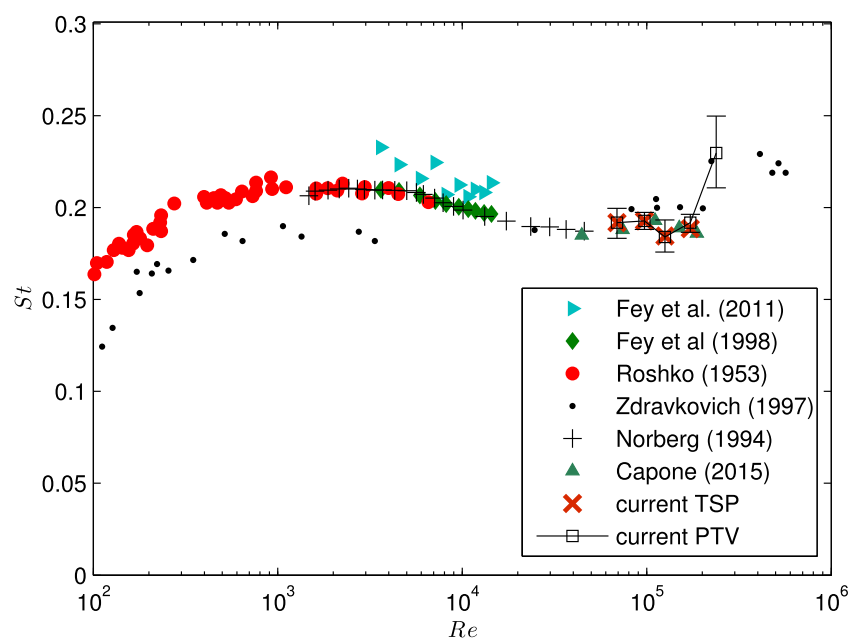

FIG. 19. The strouhal number obtained as per spectral data from Figs. 14 and 17 is plotted as a function of the Reynolds number for the current experiment and reference data. 
as reported by other authors (Bearman, 1969 among others). The ratio of the peak values of the normalized spectra obtained from TSP to those calculated from PTV (not shown) confirms that the drop in the shedding frequency peak strength as calculated from the separation line anticipates the onset of the critical regime. The analogous results discussed in Fig. 11, where the line of skin friction convergence associated with the final flow detachment anticipates the behavior of the laminar separation, suggest that prior to entering the critical regime a flow state occurs, which is marked by a not fully reattached boundary layer, reduced strength of the spectrum peak of the oscillations of the angle of laminar separation, and a delay of the flow detachment from the cylinder surface, whereas the structure of the near-wake shear layers is still typical of a sub-critical state and the shedding is still regular.

\section{CONCLUSIONS}

A complex scenario as the flow undergoes transition from sub-critical to critical regime is revealed by combined results from near-wake velocimetry data, surface temperature measurements, and relative skin friction topology, with the latter providing a valuable insight principally under conditions characterized by strong velocity gradients. In particular, the fast response time of the TSP coating employed and the inherent capability to produce surface data make it possible to attain a broader perspective than that obtained from single-point measurements, usually employed in the literature works on the subject. Near-wake measurements show dramatic flow modifications as to the structure of the shear layers. In the critical regime, they close up to each other giving rise to wake narrowing as well as the ending of regular shedding. The analysis of skin friction lines of convergence/divergence along with the temperature profiles shows the boundary layer transition to turbulence and how the increase of the final flow separation angle anticipates the laminar separation angle rise reported at critical Reynolds numbers. In the critical regime, the increase of the mixed turbulent stress in the near-wall region matches the region of turbulent reattachment line in relative skin friction maps, emphasizing the link between boundary layer transition to turbulence and shear-layer instability. Power spectral data show, in the sub-critical regime, strong peaks associated with the common vortex shedding frequency both in near-wake and surface measurements with good match to Strouhal number data. Reynolds mixed stress results confirm that at this flow regime, the shear layers develop relatively far from the wall surface. The twofold perspective provided by the TSP-PTV setup sheds light on the alterations of the shedding mechanism for increasing Reynolds number which translates into different behaviors onto the surface and in the near-wake. As the Reynolds number approaches the critical regime, the increasingly energetic boundary layer is supposedly less sensitive to the flow fluctuations and, as a consequence, the associated line of flow separation on the cylinder surface displays a weak oscillating pattern. The simultaneity of TSP and PTV acquisitions allows calculation of the correlation coefficient of the separation angle time history and local vorticity time series. Observation of correlation maps confirms that as the flow approaches the critical regime, surface and wake oscillations become increasingly disconnected. In the critical regime, the contraction of the high-correlation region and the weakening of surface oscillations suggest the onset of a different shedding mechanism acting no more on the cylinder surface.

\section{ACKNOWLEDGMENTS}

This work has been supported by the Flagship Project RITMARE, The Italian Research for the Sea, coordinated by the Italian National Research Council, and funded by the Italian Ministry of Education, University and Research.

Achenbach, E., "Distribution of local pressure and skin friction around a circular cylinder in cross-flow up to $r e=5 \times 106$," J. Fluid Mech. 34, 625-639 (1968).

Achenbach, E., "Total and local heat transfer from a smooth circular cylinder in cross-flow at high Reynolds number," Int. J. Heat Mass Transfer 18, 1387-1396 (1975).

Arie, M., Kiya, M., Suzuki, Y., Hagino, M., and Takahashi, K., "Characteristics of circular cylinders in turbulent flows," Bull. JSME 24, 640-647 (1981). 
Bearman, P., "On vortex shedding from a circular cylinder in the critical Reynolds number regime,” J. Fluid Mech. 37, 577-585 (1969).

Cantwell, B. and Coles, D., "An experimental study of entrainment and transport in the turbulent near wake of a circular cylinder,” J. Fluid Mech. 136, 321-374 (1983).

Capone, A., Klein, C., Di Felice, F., Beifuss, U., and Miozzi, M., "Fast-response underwater TSP investigation of subcritical instabilities of a cylinder in crossflow," Exp. Fluids 56, 1-14 (2015).

Djeridi, H., Braza, M., Perrin, R., Harran, G., Cid, E., and Cazin, S., "Near-wake turbulence properties around a circular cylinder at high Reynolds number," Flow, Turbul. Combust. 71, 19-34 (2003).

Fey, U., Egami, Y., and Engler, R. H., "High Reynolds number transition detection by means of temperature sensitive paint," in 44th AIAA Aerospace Sciences Meeting and Exhibit (AIAA, Reno, NV, 2006), pp. 2006-514.

Fey, U., Klein, C., Möller, T., Pöttner, J., Radespiel, R., Ondrus, V., and Beifuß, U., "Investigation of circular cylinder flow in water using temperature-sensitive paint," in New Results in Numerical and Experimental Fluid Mechanics VIII (Springer, 2013), pp. 657-664.

Horn, B. K. and Schunck, B. G., "Determining optical flow," Artif. Intell. 17, 185-203 (1981).

Kiya, M., Suzuki, Y., Arie, M., and Hagino, M., "A contribution to the free-stream turbulence effect on the flow past a circular cylinder," J. Fluid Mech. 115, 151-164 (1982).

Klein, C., Henne, U., Sachs, W., Beifuss, U., Ondrus, V., Bruse, M., Lesjak, R., Löhr, M., Becher, A., and Zhai, J., "Combination of temperature-sensitive paint (TSP) and carbon nanotubes (CNT) for transition detection," in 53rd AIAA Aerospace Sciences Meeting (American Institute of Aeronautics and Astronautics, 2015).

Kwok, K. C., "Turbulence effect on flow around circular cylinder," J. Eng. Mech. 112, 1181-1197 (1986).

Liu, T., Pressure-and Temperature-Sensitive Paints (Wiley Online Library, 2004).

Liu, T., "Extraction of skin-friction fields from surface flow visualizations as an inverse problem," Meas. Sci. Technol. 24, 124004 (2013).

Liu, T. and Woodiga, S., "Feasibility of global skin friction diagnostics using temperature sensitive paint," Meas. Sci. Technol. 22, 115402 (2011).

Lucas, B. D., Kanade, T. et al., "An iterative image registration technique with an application to stereo vision," in International Joint Conference on Artificial Intelligence (1981), Vol. 81, pp. 674-679.

Miau, J., Tsai, H., Lin, Y., Tu, J., Fang, C., and Chen, M., "Experiment on smooth, circular cylinders in cross-flow in the critical Reynolds number regime," Exp. Fluids 51, 949-967 (2011).

Miozzi, M., "Particle image velocimetry using feature tracking and delaunay tessellation," in Proceedings of the XII International Symposium on Application of Laser Technique to Fluid Mechanics, Lisbon (2004).

Miozzi, M., "Accuracy of $\mathrm{C} 1$ natural neighbors interpolation of feature tracking data and vorticity measurements," in 6th International Symposium on Particle Image Velocimetry (Caltec, Pasadina, Ca, 2005).

Norberg, C. and Sunden, B., "Turbulence and Reynolds number effects on the flow and fluid forces on a single cylinder in cross flow," J. Fluids Struct. 1, 337-357 (1987).

Ondrus, V., Meier, R., Klein, C., Henne, U., Schferling, M., and Beifuss, U., "Europium 1,3-di(thienyl)propane-1,3-diones with outstanding properties for temperature sensing," Sens. Actuators, A 233, 434-441 (2015).

Perrin, R., Braza, M., Cid, E., Cazin, S., Chassaing, P., Mockett, C., Reimann, T., and Thiele, F., "Coherent and turbulent process analysis in the flow past a circular cylinder at high Reynolds number," J. Fluids Struct. 24, 1313-1325 (2008).

Perrin, R., Cid, E., Cazin, S., Sevrain, A., Braza, M., Moradei, F., and Harran, G., "Phase-averaged measurements of the turbulence properties in the near wake of a circular cylinder at high Reynolds number by 2c-piv and 3c-piv," Exp. Fluids 42, 93-109 (2007).

Roshko, A., "Experiments on the flow past a circular cylinder at very high Reynolds number," J. Fluid Mech. 10, 345-356 (1961).

Roshko, A., "Perspectives on bluff body aerodynamics," J. Wind Eng. Ind. Aerodyn. 49, 79-100 (1993)

Sadeh, W. Z. and Saharon, D. B., "Turbulence effect on crossflow around a circular cylinder at subcritical Reynolds numbers," Contractor Report No. 3622, NASA (1982).

Singh, S. and Mittal, S., "Flow past a cylinder: Shear layer instability and drag crisis,” Int. J. Numer. Methods Fluids 47, 75-98 (2005).

Stanislas, M., Okamoto, K., Kähler, C. J., Westerweel, J., and Scarano, F., "Main results of the third international PIV challenge," Exp. Fluids 45, 27-71 (2008).

Tropea, C., Yarin, A. L., and Foss, J. F., Springer Handbook of Experimental Fluid Mechanics (Springer Science \& Business Media, 2007), Vol. 1.

Williamson, C. H., "Vortex dynamics in the cylinder wake," Annu. Rev. Fluid Mech. 28, 477-539 (1996).

Wu, J., Tramel, R., Zhu, F., and Yin, X., “A vorticity dynamics theory of three-dimensional flow separation,” Phys. Fluids 12, 1932-1954 (2000).

Yorita, D., Asai, K., Klein, C., Henne, U., and Schaber, S., "Transition detection on rotating propeller blades by means of temperature-sensitive paint," in Proceedings of the 50th AIAA Aerospace Sciences Meeting (AIAA, 2012), p. 1187.

Zdravkovich, M., "Flow around circular cylinders. Volume I: Fundamentals," J. Fluid Mech. 350, 377-378 (1997). 